\title{
Commentary: Spinal cord injury after the frozen elephant trunk procedure: Are we really safe now?
}

\author{
Oliver J. Liakopoulos, MD
}

\author{
From the Department of Cardiothoracic Surgery, Heart Center, University of Cologne, Cologne, Germany. \\ Disclosures: Author has nothing to disclose with regard to commercial support. \\ Received for publication April 7, 2019; accepted for publication April 8, 2019; available ahead of print May 14, \\ 2019. \\ Address for reprints: Oliver J. Liakopoulos, MD, Department of Cardiothoracic Surgery, Heart Center of the \\ University of Cologne, Kerpener Strasse 62, 50924 Cologne, Germany (E-mail: oliver.liakopoulos@ \\ uk-koeln.de). \\ J Thorac Cardiovasc Surg 2020;159:1199-200 \\ $0022-5223 / \$ 36.00$ \\ Copyright (C) 2019 by The American Association for Thoracic Surgery \\ https://doi.org/10.1016/j.jtcvs.2019.04.008
}

Since its introduction in the early 1990s, the frozen elephant trunk (FET) technique has become popular for the surgical treatment of aortic arch pathologies in many centers worldwide. $^{1,2}$ The hybrid stent graft facilitates in certain pathologies a complete 1-stage repair of the aortic arch and descending aorta and provides an ideal landing zone for a 2-staged thoracic endovascular aortic repair. Consequently, the Vascular Domain of European Association of Cardiothoracic Surgery recommends the FET procedure for the treatment of certain aortic arch pathologies, including younger patients with acute type A aortic dissections (AAADs) (class II B/C recommendation). ${ }^{3}$

Despite the potential advantages of the FET technique, the clinical benefit of this modern approach compared with conventional aortic arch repair (CAR) is still controversially discussed in the absence of data from wellcontrolled, prospective trials. ${ }^{3,4}$ Chakos and coworkers ${ }^{5}$ summarized in a recent systematic literature review the results of 9 retrospective studies comparing a total of 841 FET with 1182 CAR cases. ${ }^{5}$ Pooled estimate analysis of operative mortality (FET: 7.8\% vs CAR: 10.4\%), stroke (FET: $7.2 \%$ vs CAR: $6.7 \%$ ), and long-term survival were roughly comparable between both techniques. However, the FET technique was associated with a higher rate of spinal cord injury (SCI) when compared with CAR (FET: $3.8 \%$ vs CAR: $1.4 \% ; P<.01)$.

Indeed, the deliberate use of the FET is discouraged by some surgeons, especially in patients with AAAD, based on the increasing evidence suggesting a higher rate of SCI associated with this technique. The incidence of SCI after conventional elephant trunk repair ranges from $0.6 \%$ to $3.6 \%{ }^{4}$ For the FET, the highly experienced group from Hannover reported a 5\% SCI rate in 94 patients with DeBakey type I aortic dissection who underwent a FET procedure in their center. ${ }^{6}$ Likewise, SCI occurred in $6.5 \%$ of 170 patients with AAAD in the international, multicenter E-vita OPEN-Plus registry using a nonbranched FET. ${ }^{2}$ Although

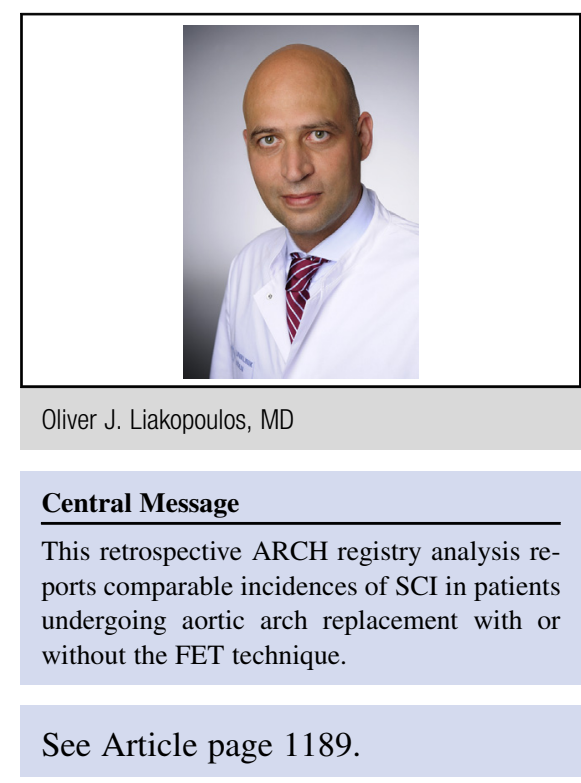

some risk factors for the observed higher incidence of SCI and its detrimental clinical sequence after the FET procedure are well established (ie, insufficient cooling of core body temperature, prolonged duration of circulatory arrest, and cerebral perfusion), others are less well understood. For example, extensive stent coverage of intercostal arteries associated with a lower distal landing zone of the FET stent graft (Th 7-10) was a predictor for SCI in some studies, 2,7 but others have failed to confirm this relation. 8,9

Poon and coworkers ${ }^{10}$ report in this issue of the Journal results from the international, multicenter $\mathrm{ARCH}$ registry and provide new evidence from the largest database analyzing this controversial topic. The investigators of the International Aortic Arch Surgery Study Group analyzed 978 patients with total arch replacement for AAAD who were entered into a registry database by 37 participating centers between 2000 and 2015. The FET technique or CAR was performed in $48 \%(\mathrm{n}=471)$ and $52 \%$ $(\mathrm{n}=507)$ of patients, respectively, and propensity score (PS) matching resulted in 149 pairs. Key end points, including operative mortality (FET: $16.1 \%$ vs CAR: $22.3 \%$ ), permanent neurologic deficit (FET: 10.1\% vs CAR $11.9 \%$ ), and, most important, the rate of SCI (FET $6.3 \%$ vs $4.0 \%$ ) did not statistically differ between the PSadjusted groups, even after hierarchical regression analysis. On the basis of the results of their study, the authors cautiously advocate that the FET technique does not 
increase the risk of SCI in appropriately selected patients and experienced centers, and that its use should be broadened to patients with AAAD.

At first glance, the data derived from this large, multiinstitutional registry seem robust enough to underscore the authors' conclusion. However, as in other registry studies, there were also several major limitations due to the retrospective design of this registry that could not be fully addressed by the authors, even by means of advanced statistical methods. Approximately 5\% of baseline and 9\% of the presented operative data were missing, and multiple data imputation was performed for variables that had at least $70 \%$ of data available. This introduces a major bias into the results of this study. Most important, a high heterogeneity remained between both surgical treatment groups despite PS adjustment, as depicted in Table 1 of the article, ${ }^{10}$ with poor matching for crucial variables, including temperature of circulatory arrest and neuroprotection strategy, indicating institutional variations of surgical techniques between surgeons and participating centers. Second, key data of risk factors known to be associated to $\mathrm{SCI}$ in patients with AAAD, including hemodynamic instability, malperfusion syndrome, preexisting neurologic disorders, and implementation of spinal fluid drainage, were not acquired by this registry. Likewise, the authors could not provide any information on the extent of the dissection or the length and level of the distal landing zone of the deployed stent graft. Consequently, both treatment arms were not matched to these potentially important variables known to be associated with SCI. Altogether, the aforementioned major limitations of the ARCH registry database limit the interpretation of the presented study results. Thus, the key questions of whether the FET procedure has comparable results to CAR with regard to SCI and which patient subgroup would benefit the most from the FET technique still remain unanswered until more robust evidence is available. Consequently, the deliberate use of the FET in patients with AAAD should be avoided, as also endorsed by existing recommendations. ${ }^{3}$

\section{References}

1. Karck M, Chavan A, Hagl C, Friedrich H, Galanski M, Haverich A. The frozen elephant trunk technique: a new treatment for thoracic aortic aneurysms. $J$ Thorac Cardiovasc Surg. 2003;125:1550-3.

2. Leontyev S, Tsagakis K, Pacini D, Di Bartolomeo R, Mohr FW, Weiss G, et al. Impact of clinical factors and surgical techniques on early outcome of patients treated with frozen elephant trunk technique by using EVITA open stent-graft: results of a multicentre study. Eur J Cardiothorac Surg. 2016;49:660-6.

3. Shrestha M, Bachet J, Bavaria J, Carrel TP, De Paulis R, Di Bartolomeo R, et al. Current status and recommendations for use of the frozen elephant trunk technique: a position paper by the Vascular Domain of EACTS. Eur J Cardiothorac Surg. 2015;47:759-69.

4. Bachet J. Open repair techniques in the aortic arch are still superior. Ann Cardiothorac Surg. 2018;7:328-44.

5. Chakos A, Jbara J, Yan TD, Tian DH. Long-term survival and related outcomes for the hybrid versus the traditional arch repair - a meta-analysis. Ann Cardiothorac Surg. 2018;7:319-27.

6. Shrestha M, Haverich A, Martens A. Total aortic arch replacement with the frozen elephant trunk procedure in acute DeBakey type I aortic dissections. Eur J Cardiothorac Surg. 2017;51(Suppl 1):i29-34.

7. Flores J, Kunihara T, Shiiya N, Yoshimoto K, Matsuzaki K, Yasuda K. Extensive deployment of the stented elephant trunk is associated with an increased risk of spinal cord injury. J Thorac Cardiovasc Surg. 2006;131:336-42.

8. Pacini D, Tsagakis K, Jakob H, Mestres CA, Armaro A, Weiss G, et al. The frozen elephant trunk for the treatment of chronic dissection of the thoracic aorta: a multicenter experience. Ann Thorac Surg. 2011;92:1663-70.

9. Leontyev S, Borger MA, Etz CD, Moz M, Seeburger J, Bakhtiary F, et al. Experience with the conventional and frozen elephant trunk techniques: a singlecentre study. Eur J Cardiothorac Surg. 2013;44:1076-82.

10. Poon SS, Tian DH, Yan T, Harrington D, Nawaytou O, Kuduvalli M, et al; on behalf of the International Aortic Arch Surgery Study Group. Frozen elephant trunk does not increase incidence of paraplegia in patients with acute type A aortic dissections. J Thorac Cardiovasc Surg. 2020;159:1189-96.e1. 